\title{
ESTUDO DE ACURÁCIA EM CIRURGIA ASSISTIDA POR NAVEGAÇÃO NA REVISÃO CIRÚRGICA DE DEFORMIDADE VERTEBRAL
}

\author{
STUDY OF THE ACCURACY OF NAVIGATION-ASSISTED SURGERY IN THE SURGICAL \\ REVISION OF SPINAL DEFORMITY \\ ESTUDIO DE LA PRECISIÓN EN CIRUGÍA ASISTIDA POR NAVEGACIÓN EN LA \\ REOPERACIÓN DE DEFORMIDAD ESPINAL \\ Chárbel Jacob Junior', Jefferson Alves Galves², Francisco Prado Eugênio dos Santos², Carlos Eduardo Algaves Soares de Oliveira ${ }^{3}$
}

RESUMO

Objetivo: Avaliar as vantagens da cirurgia com navegação na revisão cirúrgica de deformidades vertebrais, verificando a acurácia deste método. Método: Foram revisados cincos pacientes com deformidades vertebrais que tiveram intercorrências na primeira cirurgia. Esses pacientes foram submetidos a um estudo de tomografia computadorizada (TC) com cortes de $2 \mathrm{~mm}$ da coluna vertebral antes da segunda cirurgia. Nos cinco pacientes submetidos a reabordagem cirúrgica procedeu-se a instrumentação posterior com auxílio da navegação. Foram 84 parafusos pediculares implantados, sendo que 33 destes parafusos foram assistidos por computação. A navegação foi empregada nos níveis da deformidade vertebral onde a anatomia apresentava-se alterada inviabilizando o correto uso dos parâmetros anatômicos para inserção de parafusos pediculares. Nos demais níveis onde era possível a correta identificação desses parâmetros anatômicos foi utilizada a técnica padrão. A TC pós-operatória foi realizada para aferição do posicionamento dos parafusos pediculares. Avaliamos os resultados obtidos no posicionamento com e sem o uso da navegação. O tempo de fluoroscopia e o tempo da cirurgia também foram comparados com o padrão ouro da literatura. Resultados: Dos 33 parafusos implantados com navegação observou-se uma acurácia de 94\%, com uma taxa de violação pedicular de 6\%. Conclusões: O uso da navegação é importante nas revisões cirúrgicas das deformidades vertebrais com anatomia alterada, influenciando no bom resultado final da cirurgia.

Descritores: Cirurgia assistida por computação; Reoperação; Escoliose.

\begin{abstract}
Objective: To evaluate the benefits of computer-assisted spine surgery in re-operations due to vertebral deformities, and verify method accuracy. Method: A total of five patients with vertebral deformities who had complications during prior surgery were re-operated. These patients underwent a specific Computer Tomography scan with $2 \mathrm{~mm}$ cuts of the spine before the second surgery. In the five patients who underwent re-operation, posterior instrumentation with the aid of computer-assisted spine surgery was performed. 84 pedicle screws were implanted, and in 33 of these computer-assisted surgeries were used. The computer-assisted surgery was used at the levels of spinal deformity where anatomy was altered making correct use of anatomical parameters for insertion of pedicle screws impossible. In the remaining levels, standard technique was used where the correct identification of anatomical parameters was possible. Post-operative computed tomography was performed to measure the placement of pedicle screws. We evaluated the results in positioning with and without the use of navigation. The fluoroscopy and surgery time were also compared with the gold standard of literature. Results: Of the 33 screws implanted with navigation, 94\% accuracy was observed; with a pedicle violation rate of $6 \%$. The use of navigation is an important tool for surgical revision of the vertebral deformities with altered anatomy, influencing surgery result.
\end{abstract}

Keywords: Surgery, computer-assisted; Re-operation; Scoliosis.

\section{RESUMEN}

Objetivo: Evaluar las ventajas de la cirugía con navegación en reoperación por deformidades vertebrales, verificando la precisión de este método. Método: Fueron revisados cinco pacientes con deformidades vertebrales que tuvieron complicaciones en la primera cirugía. Esos pacientes fueron sometidos a un estudio de tomografía computada (TC) con cortes de $2 \mathrm{~mm}$ de la columna vertebral antes de la segunda cirugía. En los 5 pacientes sometidos al reabordaje quirúrgico se procedió a instrumentación posterior con auxilio de la navegación. Fueron 84 tornillos pediculares implantados, siendo que 33 de éstos fueron asistidos por computación. La navegación fue empleada en los niveles de la deformidad vertebral en donde la anatomía se presentaba alterada impidiendo el uso correcto de los parámetros anatómicos para inserción de tornillos pediculares. En los demás niveles en donde era posible la correcta identificación de esos parámetros anatómicos se utilizó la técnica estándar. La TC postoperatoria fue realizada para medición del posicionamiento de los tornillos pediculares. Evaluamos los resultados obtenidos en el posicionamiento con y sin el uso de la navegación. El tiempo de fluoroscopía y el tiempo de la cirugía también fueron comparados con la regla áurea de la literatura. Resultados. De los 33 tornillos implantados con navegación se observó una precisión de 94\%, con una tasa de violación pedicular del 6\%. Conclusiones: El uso de la navegación es importante en la reoperación de las deformidades vertebrales con anatomía alterada, influenciando en el buen resultado de la cirugía.

Descriptores: Cirugía asistida por computación; Reoperación; Escoliosis.

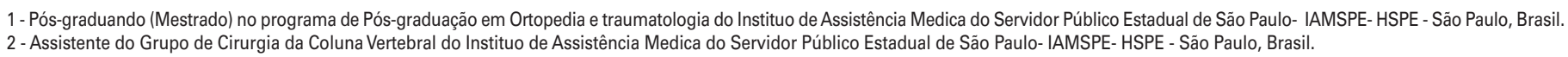

3 - Chefe do Grupo de Cirurgia da Coluna Vertebral do Instituto de Assistência Medica do Servidor Público Estadual de São Paulo- IAMSPE- HSPE, - São Paulo, Brasil.

Trabalho realizado no Setor de Ortopedia e Traumatologia do Instituto de Assistência Medica do Servidor Público Estadual de São Paulo -IAMSPE.

Correspondência: Rua Ceará n 10 - apto 1004 - Praia da Costa - Vila Velha - Brasil - 29101290 - E-mail: jcharbel@ gmail.com

Recebido em 28/06/2010, aceito em 24/10/2010. 


\section{INTRODUÇÃO}

O tratamento da deformidade da coluna vertebral depende das características clínicas e radiográficas, como o equilíbrio espinopélvico sagital e coronal, assim como a magnitude da curva e o potencial de crescimento da mesma.

O tratamento cirúrgico da escoliose é realizado com o instrumental de terceira geração na maioria dos casos, permitindo correção da curva nos três planos, restaurando o equilíbrio espinopélvico, e prevenindo a progressão da deformidade ${ }^{1,2}$. No entanto, as complicações do tratamento cirúrgico já estão bem definidas. Estudo recente revelou que 9\% dos pacientes operados por deformidades são submetidos à pelo menos uma revisão cirúrgica, sendo que $1,5 \%$ são submetidos a mais que uma revisão ${ }^{3}$. As razões mais comuns são: pseudo-artrose $(41,4 \%)$, progressão da curva $(20,7 \%)$, infecção $(1,5 \%)$ e mais raramente o déficit neurológico $(0,2 \%)^{3-6}$

A fixação pedicular tem uma série de vantagens para correção das deformidades em relação aos outros métodos de fixação da coluna vertebral, porém o potencial das complicações são mais graves $^{4-9}$. O uso da fluoroscopia no auxílio da fixação das deformidades vertebrais têm acurácia relativa, em especial, nas reabordagens de escoliose, podendo ter altas taxas de violação pedicular ${ }^{10-12}$.

A necessidade de um melhor posicionamento dos parafusos pediculares nas reabordagens da escoliose levou a investigação e aplicação do auxílio da navegação em cirurgias da coluna vertebral $^{13-18}$

A navegação, de inicio, foi utilizada por Reinhart e Barned em 1993, na neurocirurgia, somente depois aplicada à cirurgia da coluna vertebral, nos pedículos lombares, torácico e cervical. A cirurgia assistida por computação representa um avanço tecnológico e científico, proporcionando mais segurança e melhores resultados, principalmente, nas cirurgias complexas ${ }^{19}$.

Nosso estudo tem como objetivo demonstrar as vantagens do auxílio da cirurgia com navegação em reabordagens de deformidades espinais, verificando a acurácia deste método.

\section{MÉTODO}

No Hospital do Servidor Público Estadual de São Paulo (HSPEIAMSPE) foram reoperados cinco pacientes com deformidades vertebrais, os quais tiveram intercorrências na cirurgia inicial. Esses pacientes foram submetidos no pré-operatório, a tomografias helicoidais de coluna total (cortes de $2 \mathrm{~mm}$ ). Durante o planejamento pré-operatório, a anatomia das vértebras a serem instrumentadas foi avaliada usando um software especifico (BRAINLAB ${ }^{\circledR}$ Iplan Spine 2.0.1), que ilustra os cortes de TC em quatro diferentes formas de visão: axial, sagital, coronal e reconstrução 3D. Além da possibilidade de verificar o tamanho e diâmetro dos parafusos que serão colocados, marcações em estruturas anatômicas foram feitas nas vértebras para registro por pontos pareados posteriormente. No intra-operatório, após a exposição vertebral, uma garra que sustenta o refletor para a navegação é fixada no processo espinhoso da vértebra de interesse ou, quando não há processo espinhoso, fixa-se na vértebra mais próxima da instrumentação (Figura 1). 0 registro é feito com um probe específico, tocando as estruturas anatômicas definidas na etapa de planejamento (Figura 2). O sistema de navegação calcula se esses pontos captados são idênticos aos planejados e, a partir desse calculo, exibe a precisão (Tabela 1).

Os instrumentais necessários para a colocação dos parafusos são calibrados e sua trajetória e ponta são exibidos na tela do navegador, tais como: AWL, probes retos e curvos, machos e chaves para o parafuso.

Os cinco casos foram submetidos à revisão cirúrgica em es-

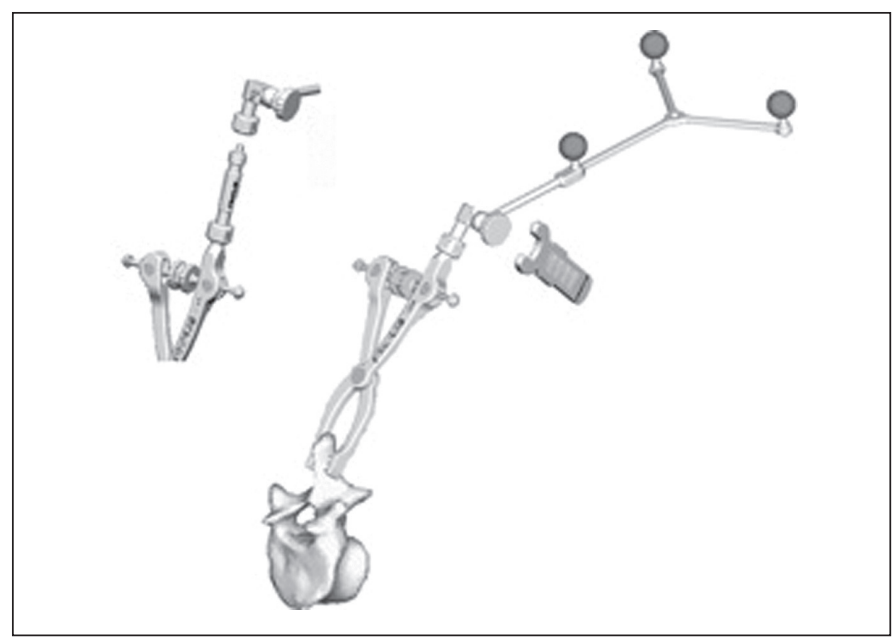

Figura 1. Garra que sustenta o refletor para a navegação é fixada no processo espinhoso da vértebra de interesse junto com a estrela de referência.

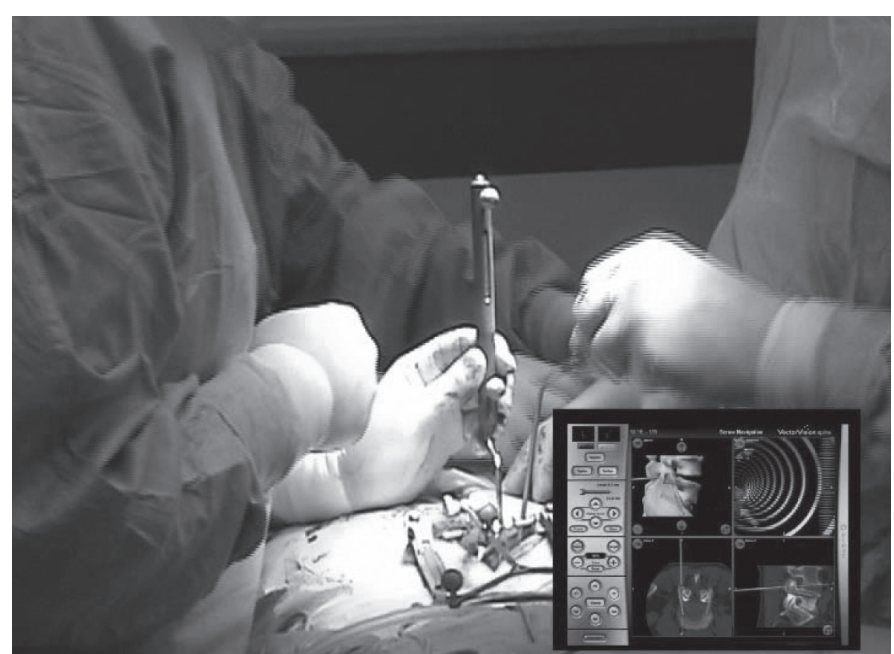

Figura 2. Probe específico tocando as estruturas anatômicas definidas na etapa de planejamento.

colioses, utilizando instrumentação por via posterior com auxílio da navegação cirúrgica (BRAINLAB Vector Vision ${ }^{\circledR}$ ) em duas deformidades do adulto, uma congênita, uma secundária a tumor intramedular, uma neuromuscular. Foram utilizados 84 parafusos pediculares, sendo 33 parafusos implantados assistido por computação (Tabela 2). Os critérios para utilização da navegação foram os principais níveis das deformidades vertebrais que estavam totalmente distorcidos, inviabilizando parâmetros anatômicos, sendo nos demais níveis utilizada a técnica habitual (Figura 3).

A TC pós-operatória foi realizada para aferição do posicionamento dos parafusos pediculares. Avaliamos os resultados obtidos no posicionamento com o uso da navegação. A TC era analisada visualizando a integridade da parede do pedículo em seus aspectos medial, lateral, inferior e superior. A posição do parafuso era graduada em milímetros na TC digitalizada, conforme a classificação de $\mathrm{RaO}^{20}$, que dividiu de acordo com o desvio lateral ou medial à parede do pedículo: tipo $A$, sem violação do pedículo, tipo $B$ somente a alma do parafuso fora do pedículo, menor que $2 \mathrm{~mm}$, tipo C metade do diâmetro do parafuso fora do pedículo, 2-4mm, tipo $D$ todo diâmetro fora do pedículo, maior que $4 \mathrm{~mm}$. Segundo a literatura, rupturas da parede lateral do pedículo de até $4 \mathrm{~mm}$ e rupturas da parede medial do pedículo até $2 \mathrm{~mm}$ são consideradas dentro da zona de segurança ${ }^{21,22}$ (Figura 4). 
Tabela 1. Relação da precisão de erro do navegador (medida em mm) em relação a cada nível vertebral de cada deformidade.

\begin{tabular}{|c|c|c|c|c|c|}
\hline & $\begin{array}{l}\text { Escoliose } \\
\text { Congênita }\end{array}$ & $\begin{array}{c}\text { Escoliose } \\
\text { degenerativa }\end{array}$ & $\begin{array}{l}\text { Escoliose } \\
\text { Idiopática* }\end{array}$ & $\begin{array}{l}\text { Escoliose } \\
2^{\mathrm{a}} \text { a TU }\end{array}$ & $\begin{array}{l}\text { Escoliose } \\
\text { Idiopática** }\end{array}$ \\
\hline $\mathrm{T} 1$ & & & & $1.2 \mathrm{~mm}$ & \\
\hline T2 & & & & $1.2 \mathrm{~mm}$ & \\
\hline T3 & $0.5 \mathrm{~mm}$ & & & $1.2 \mathrm{~mm}$ & \\
\hline T4 & $0.5 \mathrm{~mm}$ & & $1.0 \mathrm{~mm}$ & & $1.0 \mathrm{~mm}$ \\
\hline T5 & $0.5 \mathrm{~mm}$ & & $1.0 \mathrm{~mm}$ & $1.0 \mathrm{~mm}$ & $1.0 \mathrm{~mm}$ \\
\hline T6 & $0.5 \mathrm{~mm}$ & & & $1.2 \mathrm{~mm}$ & \\
\hline T7 & & & $0.5 \mathrm{~mm}$ & $1.0 \mathrm{~mm}$ & \\
\hline T8 & & & & & $2.1 \mathrm{~mm}$ \\
\hline T9 & & & & & $2.1 \mathrm{~mm}$ \\
\hline T10 & $0.9 \mathrm{~mm}$ & & & & $1.0 \mathrm{~mm}$ \\
\hline T11 & & & & $1.3 \mathrm{~mm}$ & \\
\hline T12 & & & & & \\
\hline L1 & & $0,4 \mathrm{~mm}$ & $0.4 \mathrm{~mm}$ & & $1.0 \mathrm{~mm}$ \\
\hline L2 & $0.9 \mathrm{~mm}$ & $0.4 \mathrm{~mm}$ & $0.4 \mathrm{~mm}$ & & \\
\hline L3 & $0.9 \mathrm{~mm}$ & $0.4 \mathrm{~mm}$ & & & \\
\hline L4 & & $0.4 \mathrm{~mm}$ & $0.4 \mathrm{~mm}$ & & $1.0 \mathrm{~mm}$ \\
\hline L5 & & $0.4 \mathrm{~mm}$ & & & \\
\hline S1 & & $0.4 \mathrm{~mm}$ & & & \\
\hline
\end{tabular}

*Escóliose idiopática que foi reabordada decorrente evolução da curva mesmo na vigência de artrodese

**Escoliose idiopática que foi reabordada decorrente choque medular na primeira cirurgia.

O tempo de fluoroscopia e o tempo da cirurgia também foram avaliados, comparando com a literatura.

O projeto de pesquisa e sua metodologia científica tiveram aprovação pelo comitê de ética do hospital em questão.

\section{RESULTADOS}

Um total de 84 parafusos pediculares foi inserido, sendo que 33 parafusos foram assistidos pela navegação, dentre os quais: foram 13 parafusos nos pacientes com escoliose idiopática, sete parafusos no paciente com escoliose por tumor intramedular, sete parafusos no paciente com escoliose congênita (Figuras 5, 6 e 7) e seis parafusos nos pacientes com escoliose degenerativa. Desses parafusos pediculares implantados com auxílio da navegação, 31
Tabela 2. Análise das deformidades, intercorrência pós operatória, quantidades de parafusos pediculares assistidos por navegação na reabordagem cirúrgica.

\begin{tabular}{c|c|c|c|c|c|c|c}
\hline Escoliose & $\begin{array}{c}1 \text { cirurgia } \\
\text { (Intercorrência) }\end{array}$ & Idade & Sexo & $\begin{array}{c}\hat{A} . \text { Cobb } \\
\text { Pré }\end{array}$ & $\begin{array}{c}\hat{A} \text { Cobb } \\
\text { Pós }\end{array}$ & $\begin{array}{c}\text { Parafusos } \\
\text { Navegados }\end{array}$ & $\begin{array}{c}\text { Parafusos } \\
\text { não } \\
\text { Navegados }\end{array}$ \\
\hline 1 Congênita & $\begin{array}{c}\text { Evolução da } \\
\text { curva }\end{array}$ & 14 & $\mathrm{M}$ & 58 & 21 & 7 & 11 \\
\hline 2 Degenerativa & Pós infecção & 56 & $\mathrm{M}$ & 45 & 23 & 6 & 9 \\
\hline $\begin{array}{c}3 \\
\text { Idiopática }\end{array}$ & $\begin{array}{c}\text { Choque } \\
\text { medular }\end{array}$ & 32 & $\mathrm{~F}$ & 72 & 31 & 7 & 10 \\
\hline 4 TU* & $\begin{array}{c}\text { Evolução da } \\
\text { curva }\end{array}$ & 16 & $\mathrm{~F}$ & 83 & 26 & 7 & 11 \\
\hline 5 Idiopática & $\begin{array}{c}\text { Evolução da } \\
\text { curva }\end{array}$ & 15 & $\mathrm{~F}$ & 52 & 20 & 6 & 10 \\
\hline TOTAL & & & & & & 33 & 51 \\
\hline
\end{tabular}

*TU-Tumor intramedular, escoliose secundária a TU.
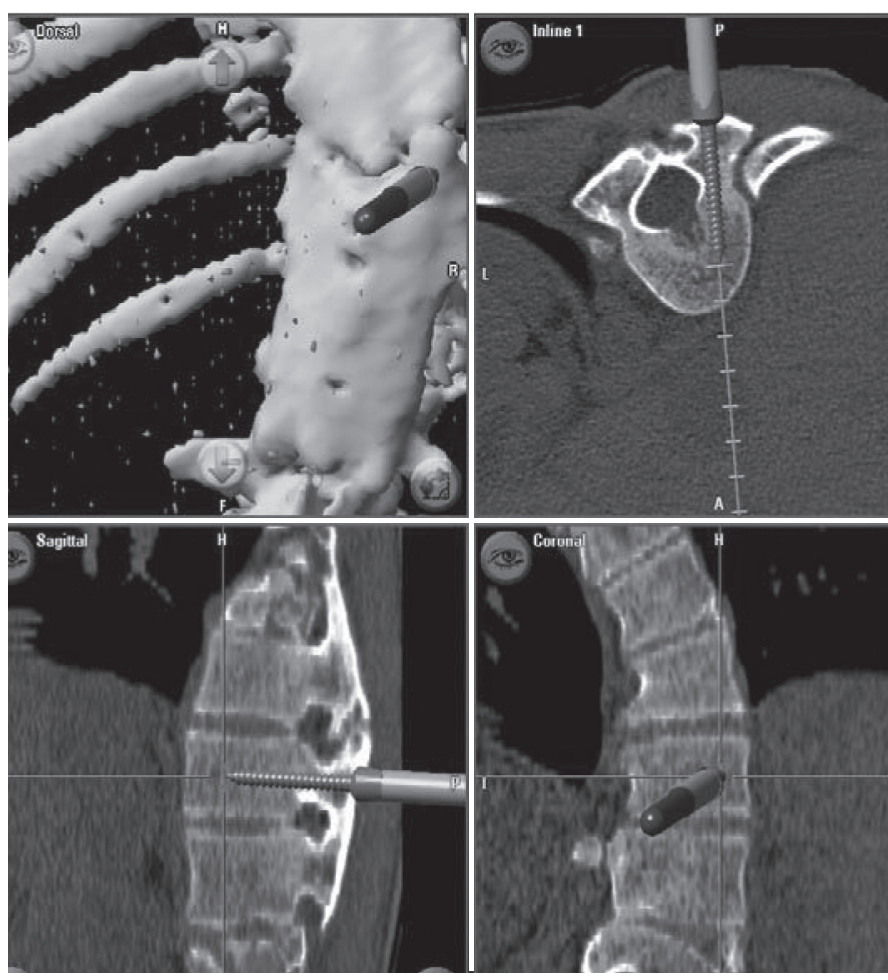

Figura 3. Principais níveis da deformidade vertebral que estavam distorcidos, inviabilizando parâmetros anatômicos.

parafusos foram implantados sem nenhuma violação do pedículo (Tipo A de Rao), todos colocados transcirúrgico com uma precisão de erro menor que $1.3 \mathrm{~mm}$, porém dois parafusos utilizados na escoliose idiopática, com uma precisão de erro de $2.1 \mathrm{~mm}$ obtiveram violação pedicular, um parafuso com desvio lateral do pedículo de $3 \mathrm{~mm}$ (grau C de Rao) e outro com violação da parede medial $2 \mathrm{~mm}$ (grau C de Rao), ambos sem repercussão clínica (Tabela 3).

O tempo cirúrgico foi de 340 minutos para revisão da escoliose secundária a tumor intramedular, média de 310 minutos para revisão das escolioses idiopáticas, 260 minutos para revisão da escoliose degenerativa, 220 minutos para revisão da escoliose Congênita, tendo uma média total de 312 minutos.

A utilização da fluoroscopia ocorreu em um nível em um caso de escoliose idiopática, decorrente do choque medular da primeira cirurgia. 

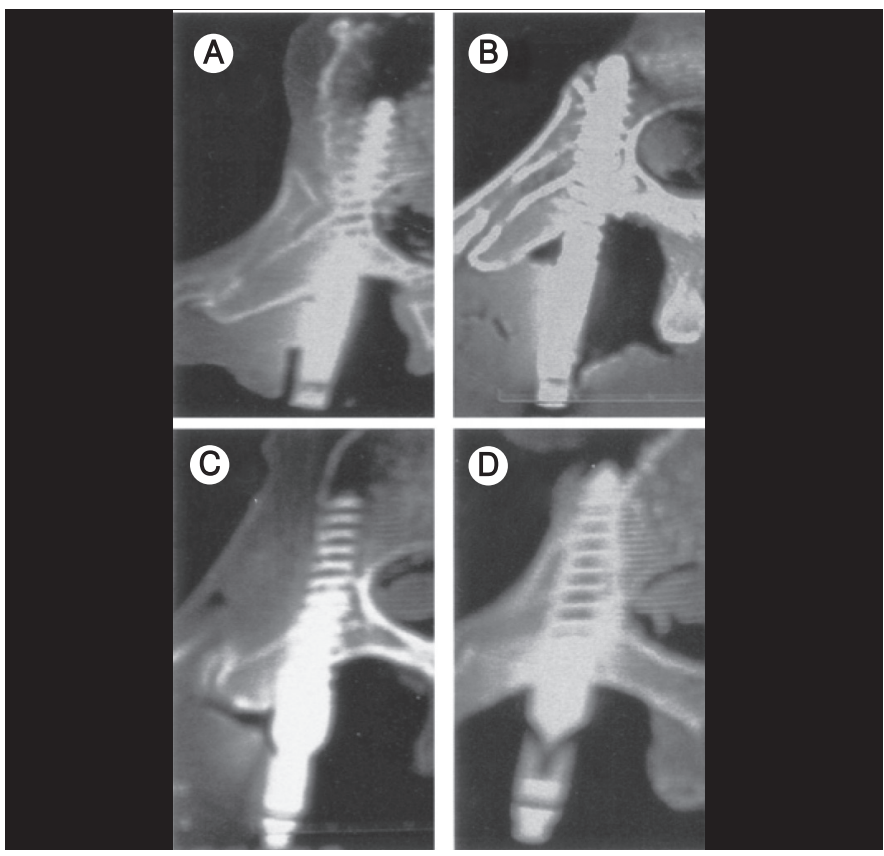

Figura 4. Sistema de Classificação de Rao et al. ${ }^{20}$, para mensurar na tomografia computadorizada o grau de violação do parafuso em relação as paredes pediculares.

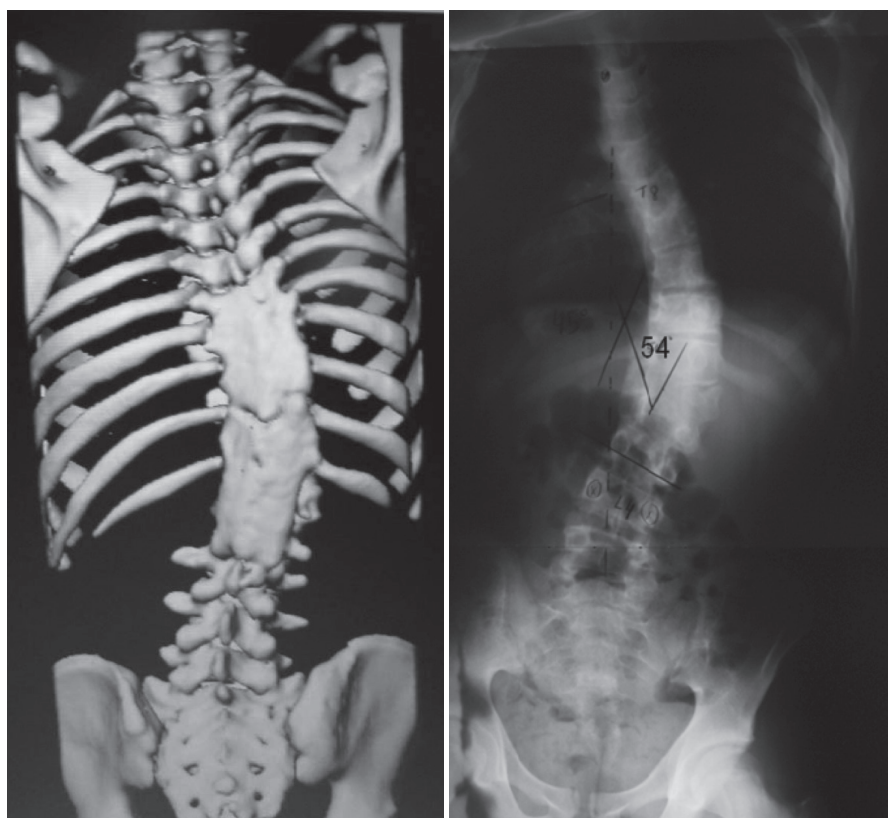

Figuras 5 e 6 . Análise pré-operatória com radiografia e TC tridimensional.

\section{ANÁLISE ESTATÍSTICA}

Houve significância estatística entre a precisão de erro do navegador intra-operatório com a localização dos parafusos pediculares colocados nos níveis com a anatomia totalmente alterada $(p=0.031)$. Demonstrando a necessidade de uma precisão de erro menor de $1,5 \mathrm{~mm}(\mathrm{P}<0,05)$, para obter sucesso na introdução dos parafusos, sem violação pedicular.

Para análise estatística utilizou-se o teste não paramétrico de Fisher.

\section{DISCUSSÃO}

A taxa de violação de parafusos pediculares colocados em "free hand" nas deformidades é de 7,9\%, segundo Kim et al. ${ }^{14}$, enquanto

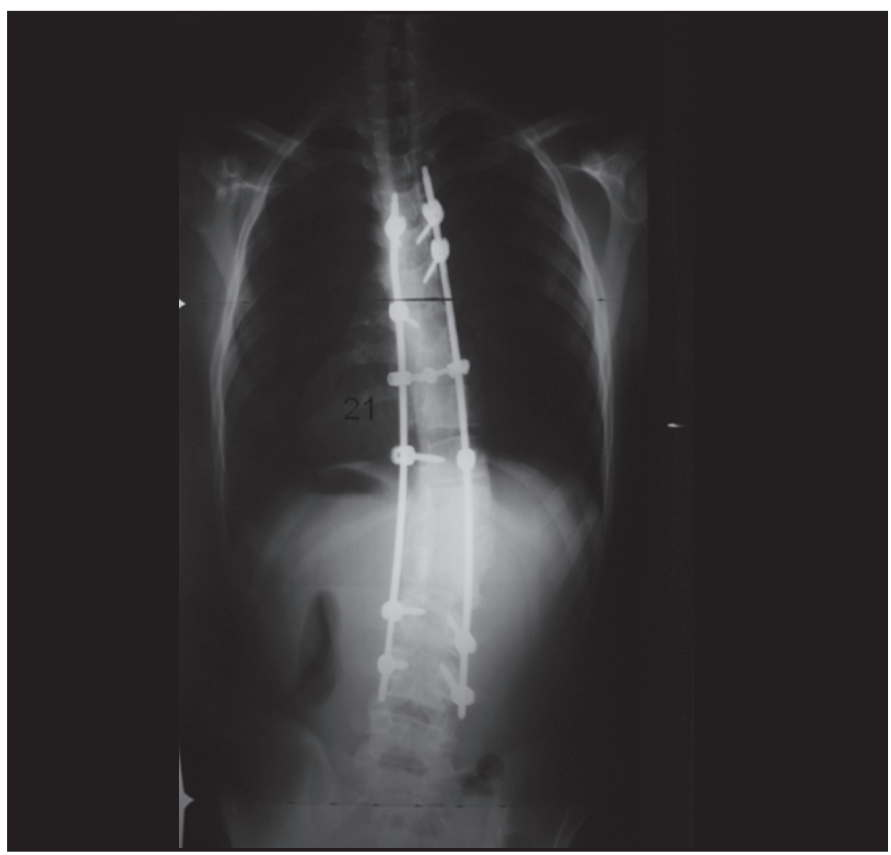

Figura 7. Pós-operatório da reabordagem da escoliose Congênita com auxílio da navegação.

Tabela 3. Relação da média da precisão de erro intra-operatória com o posicionamento do parafuso pedicular pela Classificação de Rao*

\begin{tabular}{|c|c|c|c|}
\hline \multirow{2}{*}{$\begin{array}{c}\text { Nível } \\
\text { T1 } \\
\end{array}$} & \multirow{2}{*}{\begin{tabular}{|c|}
$\begin{array}{c}\text { Precisão do Navegador } \\
\text { Intra-operatória }\end{array}$ \\
1,2 \\
\end{tabular}} & \multicolumn{2}{|c|}{$\begin{array}{l}\text { Posicionamento do parafuso pedicular } \\
\text { (Classificação de Rao) }\end{array}$} \\
\hline & & Tipo A & sem violação pedicular \\
\hline $\mathrm{T} 2$ & 1,2 & Tipo A & sem violação pedicular \\
\hline T3 & 1,2 & Tipo A & sem violação pedicular \\
\hline T4 & 1,0 & Tipo A & sem violação pedicular \\
\hline T5 & 0,75 & Tipo A & sem violação pedicula \\
\hline T6 & 0,85 & Tipo A & sem violação pedicular \\
\hline $\mathrm{T7}$ & 1,0 & Tipo A & sem violação pedicular \\
\hline T8 & 2,1 & Tipo C & Desvio $2 \mathrm{~mm}$ medial \\
\hline T9 & 2,1 & Tipo C & Desvio $2 \mathrm{~mm}$ medial \\
\hline $\mathrm{T} 10$ & 1,0 & Tipo A & sem violação pedicular \\
\hline $\mathrm{T} 11$ & 1,1 & Tipo A & sem violação pedicular \\
\hline \multicolumn{4}{|l|}{$\mathrm{T} 12$} \\
\hline $\mathrm{L} 1$ & 0,9 & Tipo A & sem violação pedicular \\
\hline L2 & 0,4 & Tipo A & sem violação pedicular \\
\hline L3 & 0,75 & Tipo A & sem violação pedicular \\
\hline L4 & 0,9 & Tipo A & sem violação pedicular \\
\hline $\mathrm{L} 5$ & 0,4 & Tipo A & sem violação pedicular \\
\hline S1 & 0,4 & Tipo A & sem violação pedicular \\
\hline
\end{tabular}

*Classificação de Rao20 (Desvio Lateral/medial do parafuso pedicular): Tipo A, sem desvio; Tipo B, <2 mm; Tipo C, 2-4mm; Tipo D, > 4 mm.

nos estudo de Halm et al. ${ }^{23}$ chega até 18,3\%. No trabalho de Lilijenqvist et al. ${ }^{24}$. a taxa de violação dos pedículos auxiliados com uso de fluoroscopia era de $25 \%$

Segundo a literatura as taxas de violação pedicular nas deformidades vertebrais variam entre 1,8\% a 11,4\% quando colocados com auxilio da navegação, dependendo da margem de erro captada pelo navegador no momento da instrumentação, tornando-se necessário uma precisão de erro do navegador menor que 1,5 mm²5.

O sistema de navegação possibilita aquisição de dados e trans- 
fere automaticamente para a interface do programa de navegação. Os segmentos vertebrais com um espaço de $12 \mathrm{~cm}^{3}$ (geralmente 5-6 níveis vertebrais na criança e 4-5 níveis vertebrais no adulto) são adquiridos durante o processo de registro. $\mathrm{O}$ uso da navegação apresentou bons resultados in vivo e em estudos laboratoriais com cadáver anteriormente ${ }^{26-28}$. Melhores resultados foram observados de maneira uniforme com o uso de navegação 22,25,27,29-30. Porém o software do navegador depende de dados da TC pré-operatória, podendo ter variação no posicionamento da coluna no intra-operatório, logo há necessidade de calibragem da superfície de cada vértebra para aumentar a acurácia do método.

Nosso trabalho apresentou uma taxa de violação pedicular de $6,06 \%$ em revisões cirúrgicas de deformidades vertebrais, semeIhante o que a literatura apresenta em casos de cirurgias primárias de deformidades ${ }^{30}$. Devem ser colocados em consideração o tipo de deformidade reoperada, e a margem de erro captada pelo navegador intra-operatorio, pois relaciona direto com a taxa de sucesso da precisão do parafuso pedicular.
Takahashi29 ${ }^{29}$ relatou em seu trabalho que o tempo médio de cirurgia foi igual ao da cirurgia não navegada, e não houve utilização da fluoroscopia durante as cirurgias navegadas. Em nosso trabalho houve um tempo cirúrgico médio de 312 minutos, semelhantes ao das cirurgias não navegadas. Houve uma redução significativa do uso da fluoroscopia em nossas cirurgias assistidas por navegação, semelhante ao da literatura.

\section{CONCLUSÃO}

A navegação demonstrou aumento da acurácia no posicionamento dos parafusos pediculares daqueles que estavam com anatomia alterada, e diminuição da exposição à radiação em cirurgias de revisão da escoliose, demonstrando ser mais segura a colocação de implantes em pedículos com anatomia alterada, sendo dependente do índice de precisão do navegador no uso intra-operatório. Restrição deve ser feita, contudo, a necessidade de um maior número de pacientes no estudo.

\section{REFERÊNCIAS}

1. Zindrick MR, Wiltse LL, Widell EH, Thomas JC, Holland WR, Field BT, et al. A biomechanical study of intrapeduncular screw fixation in the lumbosacral spine. Clin Orthop Relat Res. 1986;(203):99-112.

2. Bradford DS, Tay BK, Hu SS. Adult scoliosis: surgical indications, operative management, complications, and outcomes. Spine (Phila Pa 1976). 1999; 24(24):2617-29.

3. De Wald CJ, Stanley T. Instrumentation-related complications of multilevel fusions for adult spinal deformity patients over age 65: surgical considerations and treatment options in patients with poor bone quality. Spine (Phila Pa 1976). 2006;31(19 Suppl):S144-51.

4. Lapp MA, Bridwell KH, Lenke LG, Daniel Riew K, Linville DA, Eck KR, et al. Long-term complications in adult spinal deformity patients having combined surgery a comparison of primary to revision patients. Spine (Phila Pa 1976). 2001;26(8):973-83.

5. Pichelmann MA, Lenke LG, Bridwell KH, Good CR, O'Leary PT, Sides BA. Revision rates following primary adult spinal deformity surgery: six hundred forty-three consecutive patients followed-up to twenty-two years postoperative. Spine (Phila Pa 1976). 2010;35(2):219-26

6. Gaines RW Jr. The use of pedicle-screw internal fixation for the operative treatment of spinal disorders. J Bone Joint Surg Am. 2000;82-A(10):1458-76.

7. Baron EM, AlbertTJ. Medical complications of surgical treatment of adult spinal deformity and how to avoid them. Spine (Phila Pa 1976). 2006; 31(19Suppl):S106-18.

8. Esses SI, Sachs BL, Dreyzin V. Complications associated with the technique of pedicle screw fixation. A selected survey of ABS members. Spine (Phila Pa 1976). 1993:18(15):2231-8.

9. Schwarzenbach O, Berlemann U, Jost B, Visarius H, Arm E, Langlotz F, et al. Accuracy of computer-assisted pedicle screw placement. An in vivo computed tomography analysis. Spine (Phila Pa 1976). 1997:22(4):452-8.

10. Amiot LP, Poulin F. Computed tomography-based navigation for hip, knee, and spine surgery. Clin Orthop Relat Res. 2004;(421):77-86

11. Hart RA, Hansen BL, Shea M, Hsu F, Anderson GJ. Pedicle screw placement in the thoracic spine: a comparison of image-guided and manual techniques in cadavers. Spine (Phila Pa 1976). 2005:30(12):E326-31.

12. Assaker R, Reyns N, Vinchon M, Demondion X, Louis E. Transpedicular screw placement: image-guided versus lateral-view fluoroscopy: in vitro simulation. Spine (Phila Pa 1976). 2001;26(19):2160-4.

13. Holly LT, Foley KT. Intraoperative spinal navigation. Spine (Phila Pa 1976). 2003;28(15 Suppl):S54-61.

14. Kim KD, Patrick Johnson J, Bloch BS O, Masciopinto JE. Computer-assisted thoracic pedicle screw placement: an in vitro feasibility study. Spine (Phila Pa 1976). 2001;26(4):360-4.

15. Lavallée S, Sautot P Troccaz J, Cinquin P, Merloz P. Computer-assisted spine surgery: a technique for accurate transpedicular screw fixation using CT data and a 3-D optical localizer. J Image Guid Surg. 1995;1(1):65-73.
16. Merloz P, Tonetti J, Eid A, Faure C, Lavallee S, Troccaz J, et al. Computer assisted spine surgery. Clin Orthop Relat Res. 1997;(337):86-96.

17. Schlenzka D, Laine T, Lund T. Computer-assisted spine surgery. Eur Spine J. 2000;9(Suppl 1):S57-64.

18. Foley KT, Simon DA, Rampersaud YR. Virtual fluoroscopy: computer-assisted fluoroscopic navigation. Spine (Phila Pa 1976). 2001;26(4):347-51

19. Enchev Y. Neuronavigation: geneology, reality, and prospects. Neurosurg Focus. 2009:27(3):E11.

20. Rao G, Brodke DS, Rondina M, Bacchus K, Dailey AT. Inter- and intraobserver reliability of computed tomography in assessment of thoracic pedicle screw placement. Spine (Phila Pa 1976). 2003;28(22):2527-30

21. Van Brussel K, Vander Sloten J, Van Audekercke R, Fabry G. Internal fixation of the spine in traumatic and scoliotic cases. The potential of pedicle screws. Technol Health Care. 1996;4(4):365-84

22. Rajasekaran S, Vidyadhara S, Ramesh P, Shetty AP. Randomized clinical study to compare the accuracy of navigated and non-navigated thoracic pedicle screws in deformity correction surgeries. Spine (Phila Pa 1976). 2007; 32(2):E56-64.

23. Halm H, Niemeyer T, Link T, Lilienqvist U. Segmental pedicle screw instrumentation in Idiopathic thoracolumbar and lumbar scoliosis. Eur. Spine J. 2000 Jun;9(3):191-7.

24. Liljenqvist UR, Halm H, Link T. Pedicle screw instrumentation of the thoracic spine in idiopathic scoliosis. Spine.1997 Oct 1;22(19):2239-45

25. Nolte LP, Zamorano LJ, Jiang Z, Wang Q, Langlotz F, Berlemann U. Imageguided insertion of transpedicular screws. A laboratory set-up. Spine (Phila Pa 1976). 1995;20(4):497-500.

26. Rampersaud YR, Simon DA, Foley KT. Accuracy requirements for image-guided spinal pedicle screw placement. Spine (Phila Pa 1976). 200;26(4):352-9.

27. Schulze CJ, Munzinger E, Weber U. Clinical relevance of accuracy of pedicle screw placement. A computed tomographic-supported analysis. Spine (Phila Pa 1976). 1998;23(20):2215-20.

28. Rampersaud YR, Pik JH, Salonen D, Faroog S. Clinical accuracy of fluoroscopic computer-assisted pedicle screw fixation: a CT analysis. Spine (Phila Pa 1976). 2005;30(7):E183-90.

29. Takahashi J, Hirabayashi $H$, Hashidate H, Ogihara N, Kato H. Accuracy of multilevel registration in image-guided pedicle screw insertion for adolescent idiopathic scoliosis. Spine (Phila Pa 1976). 2010;35(3):347-52

30. Kotani Y, Abumi K, Ito M, Takahata M, Sudo H, Ohshima S, Minami A. Accuracy analysis of pedicle screw placement in posterior scoliosis surgery: comparison between conventional fluoroscopic and computer-assisted technique. Spine (Phila Pa 1976). 2007;32(14):1543-50. 